INTERVENTIONAL CARDIOLOGY

\title{
BMI inversely associated with bleeding and mortality after PCI
}

Increased BMI is often associated with poor health and worsening outcomes after a variety of medical interventions. However, in a retrospective analysis of patients who received percutaneous coronary intervention (PCI) for stable coronary artery disease or nonST-segment elevation acute coronary syndrome, BMI was inversely associated with bleeding and death at 1 year after the procedure.

Patients who received PCI $(n=14,178)$ were stratified according to BMI (quartile [Q]1, 14.1-24.8; Q2, >24.8-27.1; Q3, >27.1-29.8; and Q4, >29.8-56.3 kg/m²). The primary end point of all-cause mortality at 1 year occurred in 413 individuals. However, for patients with the lowest BMI (Q1), the Kaplan-Meier estimate of mortality at 1 year was $4.4 \%$ compared with $2.3 \%$ for those with the highest BMI (Q4; log-rank test $P<0.001$ ).

Furthermore, the frequency of bleeding in patients in Q1 was 13.8\% compared with only 7.7\% for patients in Q4 (OR 1.90, 95\% CI 1.63-2.23, $P<0.001$ ). Although bleeding occurred more often in women than men $(15.4 \%$ vs $9.1 \%$; $P<0.001)$, the frequency decreased with increasing BMI in both sexes $(P<0.001$ for trend in both men and women). The team calculate using Cox proportional hazard models that for each $1 \mathrm{~kg} / \mathrm{m}^{2}$ decrease in BMI the adjusted risk of bleeding or 1-year mortality increases by $5 \%(P<0.001)$ and $3 \%(P=0.048)$ respectively.

The investigators note that no interaction was seen between bleeding and BMI in predicting mortality, which might indicate that bleeding increases the risk of death regardless of BMI.

Tim Geach

Original article Ndrepepa, G. et al. Relation of body mass index to bleeding during percutaneous coronary interventions. Am. J. Cardiol. doi:10.1016/ j.amjcard.2014.11.022 